\title{
Usefulness of fecal calprotectin by monoclonal antibody testing in adult Japanese with inflammatory bowel diseases: a prospective multicenter study
}

\author{
Shiro Nakamura ${ }^{1}$, Hirotsugu Imaeda ${ }^{2}$, Hiroki Nishikawa ${ }^{3}$, Masaki Iimuro ${ }^{1}$, Minoru Matsuura ${ }^{4}$, Hideo Oka ${ }^{5}$, \\ Junsuke Oku ${ }^{5,6}$, Takako Miyazaki ${ }^{1,7}$, Hirohito Honda ${ }^{8}$, Kenji Watanabe $^{1,7}$, Hiroshi Nakase ${ }^{4,9}$, Akira Andoh ${ }^{2}$ \\ ${ }^{1}$ Division of Internal Medicine, Department of Inflammatory Bowel Disease, Hyogo College of Medicine, Nishinomiya, ${ }^{2}$ Department of Medicine, \\ Shiga University of Medical Science, Otsu, ${ }^{3}$ Center for Clinical Research and Education, Hyogo College of Medicine, Nishinomiya, ${ }^{4}$ Department \\ of Gastroenterology and Hepatology, Graduate School of Medicine Kyoto University, Kyoto, ${ }^{5}$ Division of Gastroenterology and Hepatology, \\ Amagasaki Central Hospital, Amagasaki, ${ }^{6}$ Oku Clinic, Higashiosaka, ${ }^{7}$ Department of Gastroenterology, Osaka City University Graduate School \\ of Medicine, Osaka, ${ }^{8}$ Sanyo Chemical Industries, Ltd., Kyoto, ${ }^{9}$ Department of Gastroenterology and Hepatology, Sapporo Medical University \\ School of Medicine, Sapporo, Japan
}

Background/Aims: Noninvasive objective monitoring is advantageous for optimizing treatment strategies in patients inflammatory bowel disease (IBD). Fecal calprotectin (FCP) is superior to traditional biomarkers in terms of assessing the activity in patients with IBD. However, there are the differences among several FCP assays in the dynamics of FCP. In this prospective multicenter trial, we investigated the usefulness of FCP measurements in adult Japanese patients with IBD by reliable enzyme immunoassay using a monoclonal antibody. Methods: We assessed the relationship between FCP levels and disease or endoscopic activity in patients with ulcerative colitis (UC, $\mathrm{n}=64$ ) or Crohn's disease (CD, $\mathrm{n}=46$ ) compared with healthy controls (HCs, $\mathrm{n}=64)$. Results: FCP levels in UC patients strongly correlated with the Disease Activity Index $\left(r_{s}=0.676, P<0.0001\right)$ and Mayo endoscopic subscore (MES; $\left.r_{s}=0.677, P<0.0001\right)$. FCP levels were significantly higher even in patients with inactive UC or CD compared with HCs $(P=0.0068, P<0.0001)$. The optimal cutoff value between MES 1 and 2 exhibited higher sensitivity (94.1\%). FCP levels were significantly higher in active UC patients than in inactive patients $(P<0.001)$, except those with proctitis. The Crohn's Disease Activity Index tended to correlate with the FCP level $\left(r_{s}=0.283, P=0.0565\right)$. Conclusions: Our testing method using a monoclonal antibody for FCP was well-validated and differentiated IBD patients from HCs. FCP may be a useful biomarker for objective assessment of disease activity in adult Japanese IBD patients, especially those with UC. (Intest Res 2018;16:554-562)

Key Words: Inflammatory bowel diseases; Colitis, ulcerative; Crohn disease; Fecal calprotectin; Antibodies, monoclonal

\section{INTRODUCTION}

Inflammatory bowel diseases (IBD), represented by UC and CD, are idiopathic lifelong immune-mediated diseases

Received March 10, 2018. Revised May 20, 2018.

Accepted May 21, 2018.

Correspondence to Shiro Nakamura, Division of Internal Medicine,

Department of Inflammatory Bowel Disease, Hyogo College of Medicine, 1-1

Mukogawacho, Nishinomiya 663-8501, Japan. Tel: +81-798-45-6663, Fax:

+81-798-45-6790, E-mail: ibd-im@hyo-med.ac.jp that cause chronic inflammation or ulcers in the mucosa or intestinal wall of the colon and small intestine. ${ }^{1-4}$ Recent advances in imaging modalities, including conventional ileocolonoscopy, magnetic resonance enterography, and small bowel endoscopy, facilitate the diagnosis of IBDs. ${ }^{5,6}$ In addition, multiple disease activity evaluation methods are available, such as the CDAI for CD and the Disease Activity Index (DAI) or Mayo score for UC, each of which has its merits and limitations because of their subjective approach. ${ }^{7,8}$ On the other hand, for the objective assessment, repeated ileocolo-

\footnotetext{
๑ Copyright 2018. Korean Association for the Study of Intestinal Diseases. All rights reserved.

This is an Open Access article distributed under the terms of the Creative Commons Attribution Non-Commercial License (http://creativecommons.org/licenses/by-nc/4.0)

which permits unrestricted non-commercial use, distribution, and reproduction in any medium, provided the original work is properly cited.
} 
noscopy in patients with IBD is inconvenient, invasive, and carries the risk of procedure-related complications. Colonoscopic procedures may also deteriorate the disease condition, even when IBD patients are in remission. ${ }^{9}$ Alternative noninvasive methods to objectively assess disease activity or predict mucosal status are thus required to optimize medical treatment strategies. Several recently identified fecal biomarkers may facilitate the differential diagnosis of IBD and IBS, and healthy bowel. ${ }^{10}$ Calprotectin, a zinc-binding and calcium-binding heterodimer of $36.5 \mathrm{kDa}$ that belongs to the S100 family, is one of the most promising fecal biomarkers of inflammation in the intestinal tract. ${ }^{10-13}$ Calprotectin is present on the surface of monocytes and macrophages, assisting in their recruitment to the site of inflammation. ${ }^{11-13}$ Because monocytes and macrophages are major sources of inflammatory cytokines and chemokines, they are suspected to have roles in the initiation and perpetuation of IBD. ${ }^{11-13} \mathrm{Cal}-$ protectin also has antimicrobial effects against Escherichia coli, Klebsiella sp., Staphylococcus aureus, and fungus, and is considered to defend against inflammation and bacterial infection in the intestinal tract. ${ }^{10,13}$ Fecal calprotectin (FCP) levels reflect the degree of gut inflammation according to the quantity of infiltrating inflammatory cells..$^{10,13}$

Numerous Western investigators report increased FCP levels in patients with IBD, and the association of FCP with disease severity. ${ }^{14-26}$ Limited data are available, however, regarding the usefulness of FCP measurements for Asian adults with UC or CD compared with healthy controls (HCs) during the same period in a prospective multicenter trial. ${ }^{27-30}$ The genetic background of IBD differs between Asian patients and Western patients. ${ }^{31}$ Therefore, investigation usefulness of FCP measurements in Japanese IBD patients as Asian IBD patients is clinically meaningful. While several kits for measuring FCP levels have recently appeared in the clinical setting, there is an urgent need to verify their accuracy. A previous study reported the predictive superiority of monoclonal testing of calprotectin compared to polyclonal testing, but their study subjects were Western patients. ${ }^{32}$ There are only 2 published studies by one investigator focusing on monoclonal antibody testing for FCP, however, in pediatric Japanese patients with IBD, ${ }^{33,34}$ and FCP levels differ between healthy children and adults. ${ }^{35}$ The aim of the present study was to evaluate the usefulness of FCP measurements for both UC and CD patients compared with HCs in a prospective multicenter trial, focusing primarily on the usefulness of reliable testing method using a monoclonal antibody for FCP measurement in adult Japanese IBD patients.

\section{METHODS}

\section{Study Design and Patient Eligibility}

This study was a prospective multicenter cooperative observational study. All analyzed subjects were patients diagnosed with UC or CD, or HCs who had undergone conventional ileocolonoscopic examinations after providing written informed consent to participate in the study at Hyogo College of Medicine, Shiga University of Medical Science, Kyoto University Graduate School of Medicine, Amagasaki Central Hospital, and Osaka City University Graduate School of Medicine between December 2011 and September 2013. Diagnosis of IBD was based on clinical, endoscopic, and histopathologic findings according to the diagnostic criteria of the Research Committee on Inflammatory Bowel Disease in Japan. Only patients with a definite diagnosis of IBD were included in the study; subjects with suspected IBD or IBD unclassified were not included. Exclusion criteria were as follows: (1) <20 years of age; (2) colon cancer or dysplasia; (3) polyps $\geq 5 \mathrm{~mm}$ in diameter; (4) diverticulitis; (5) treatment with NSAID. For UC patients, those with DAI score by Sutherland et al. ${ }^{7,36} \leq 2$ points were defined as having inactive UC and others were defined as having active UC The Mayo endoscopic subscoring system (MES, grade 0-3) was also used for endoscopic evaluation in patients with UC. Similarly, for CD patients, those with $\mathrm{CDAI}<150$ points were defined as having inactive $\mathrm{CD}$ and others were defined as having active $\mathrm{CD} .{ }^{7}$ We examined the relationship between FCP levels and disease activity in UC and CD patients.

All UC patients, all HCs, and some of CD patients underwent full colonoscopy with confirmed absence of inflammatory lesions, as well as esophagogastroduodenoscopy with confirmed absence of tumors and ulcers. The presence or absence of abdominal symptoms was also reported. Subjects with abdominal symptoms such as bowel movement abnormalities, abdominal pain, abdominal discomfort, diarrhea, and constipation were defined as the positive abdominal symptom group (PASG) and others were defined as the negative abdominal symptom group (NASG). The ethics committee at each participating facility approved this study protocol, which strictly adhered to all provisions of the Declaration of Helsinki.

\section{Measurement of FCP}

Fecal samples were in principle obtained within 7 days prior to endoscopic examinations. Fecal samples obtained after endoscopic examination were taken between 3 and 14 days after the endoscopic examination. No fecal samples 
from the colonoscopy preparation were used. The first fecal samples obtained on the day of sample collection were used for analysis. The obtained samples were stored at $-20^{\circ} \mathrm{C}$ or lower until the measurement. FCP values were measured using the Calprotectin ELISA Kit (Calprotectin Mochida ${ }^{\circledR}$ : BÜHLMANN Laboratories AG, Switzerland, and Mochida Pharmaceutical have sales contracts) according to the manufacturer's instructions. ${ }^{32}$ In brief, measurement of FCP levels is based on sandwich enzyme immunoassay using a monoclonal antibody. FCP levels are calculated by measuring the absorbance with a spectrophotometer. Measurement of FCP in all fecal samples was performed at Sanyo Chemical Industries, Ltd., Japan, which commercialized the Calprotectin ELISA kit.

\section{Statistical Analysis}

Continuous parameters were compared by an unpaired $t$ test or Mann-Whitney $U$-test as applicable. The relation between FCP levels and disease severity was calculated based on Spearman's rank correlation coefficient $r_{s}$. In continuous parameters, receiver operating characteristics (ROC) curve analysis was conducted to set the optimal cutoff point. The cutoff points were determined when the sum of sensitivity and specificity peaked (the best Youden index). Area under the ROC (AUC) was also calculated. Because FCP levels had logarithmic distributions, log10 (FCP level) was used for analysis.

Data are shown as the median value (range) unless otherwise mentioned. Statistical significance was set at $P<0.05$. Statistical analysis was performed with JMP 13 (SAS Institute Inc., Cary, NC, USA).

\section{RESULTS}

\section{Baseline Characteristics}

Baseline characteristics are shown in Table 1. The analysis included 64 HCs, 64 subjects with UC, and 46 subjects with CD. The median DAI score for UC patients was 3 (range, $0-12)$. Active UC patients comprised $57.8 \%$ (37/64) of all the UC patients. The median CDAI for CD patients was 123.2 (range, 17-380). Active CD patients comprised 39.1\%

Table 1. Baseline Characteristics

\begin{tabular}{|c|c|c|c|}
\hline Characteristics & $\mathrm{HC}(\mathrm{n}=64)$ & $U C(n=64)$ & $C D(n=46)$ \\
\hline Sex (male/female) & $43 / 21$ & $37 / 27$ & $38 / 8$ \\
\hline Age (yr) & $63(21-81)$ & $45.5(20-75)$ & $34.5(20-70)$ \\
\hline BMI $\left(\mathrm{kg} / \mathrm{m}^{2}\right)$ & $23.0(16.4-32.9)$ & $21.4(14.4-29.4)$ & $20.7(16.7-34.5)$ \\
\hline Abdominal symptoms in $\mathrm{HC}$ (yes/no) & $21 / 43$ & NA & NA \\
\hline Disease Activity Index (UC) & NA & $3(0-12)$ & NA \\
\hline CDAI (CD) & NA & NA & $123.2(17-380)$ \\
\hline IBD active (yes/no) & NA & $37 / 27$ & $18 / 28$ \\
\hline \multirow[t]{4}{*}{ Disease extent and location (Montreal classification [33]) } & NA & E1: 6 & L1: 16 \\
\hline & & E2: 25 & L2: 6 \\
\hline & & E3: 29 & L3: 22 \\
\hline & & Others: 4 & Others: 2 \\
\hline Red blood cell count $(/ \mu \mathrm{L})$ & $483(397-540)$ & $420(300-594)$ & $458(275-612)$ \\
\hline Hemoglobin (g/dL) & $14.8(12.7-15.8)$ & $12.4(9.8-18.5)$ & $13.0(8.1-17.4)$ \\
\hline White blood cell count $(/ \mu \mathrm{L})$ & $5,375(3,820-8,600)$ & $5,500(1,043-14,820)$ & $5,700(3,200-9,000)$ \\
\hline Platelet count $\left(\times 10^{4} / \mathrm{mm}^{3}\right)$ & $21.7(15.7-30)$ & $26.2(13.7-71.1)$ & $25.8(17.4-58.4)$ \\
\hline Total protein (g/dL) & $7.2(6.3-7.6)$ & $7.0(5.2-8.3)$ & $6.8(5.0-7.8)$ \\
\hline Serum albumin (g/dL) & $4.4(3.8-4.9)$ & $4.05(2.3-4.9)$ & $3.7(2.4-4.7)$ \\
\hline $\mathrm{CRP}(\mathrm{mg} / \mathrm{dL})$ & $0.08(0-0.12)$ & $0.1(0-10)$ & $0.2(0-5.1)$ \\
\hline $\mathrm{ESR}(\mathrm{mm} / \mathrm{hr})$ & NA & $12.0(0-81)$ & $8(0-109)$ \\
\hline Fecal calprotectin $(\mu \mathrm{g} / \mathrm{g})$ & $31.1(2.8-568.3)$ & $335.8(6.7-24,422.1)$ & $258.2(2.0-6,911.8)$ \\
\hline
\end{tabular}

Values are presented as median (range).

HC, healthy control; E1, ulcerative proctitis; E2, left-sided UC; E3, extensive UC; L1, terminal ileum; L2, colon; L3, ileocolon. 
(18/46) of all CD patients. FCP levels ranged from 2.8 to $568.3 \mu \mathrm{g} / \mathrm{g}$ (median, $31.1 \mu \mathrm{g} / \mathrm{g}$ ) in HCs, 6.7 to $24,422.1 \mu \mathrm{g} / \mathrm{g}$ (median, $335.8 \mu \mathrm{g} / \mathrm{g}$ ) in patients with $\mathrm{UC}$, and 2.0 to $6,911.8$ $\mu \mathrm{g} / \mathrm{g}$ (median, $258.2 \mu \mathrm{g} / \mathrm{g}$ ) in patients with CD. FCP levels were significantly higher in UC and CD patients than in HCs $(P<0.0001$ for UC and $P<0.0001$ for CD) (Fig. 1). The difference in the FCP level between UC and CD patients was not significant $(P=0.1393)$ (Fig. 1). With regard to disease extent as defined by the Montreal classification for UC patients, 6 patients had E1 involvement (ulcerative proctitis), 25 had E2 involvement (left-sided UC), 29 had E3 involvement (extensive UC), and 4 had other involvement. ${ }^{37}$ As for disease extent as defined by the Montreal classification for $\mathrm{CD}$ patients, 16 patients had L1 (ileum) involvement, 6 had L2 (colon)

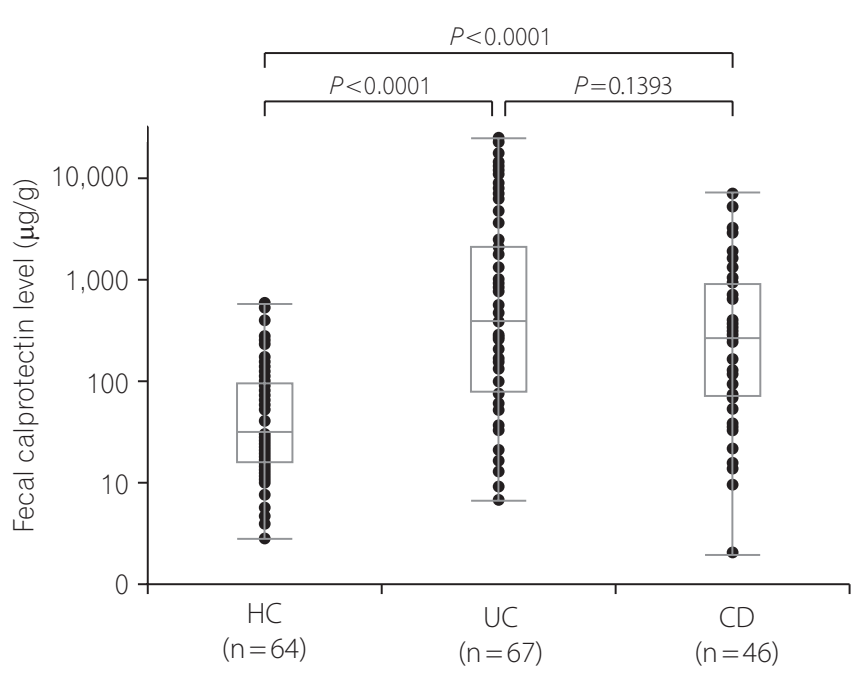

Fig. 1. Fecal calprotectin levels in healthay control $(\mathrm{HC})$ subjects, patients with UC, and patients with CD.

A

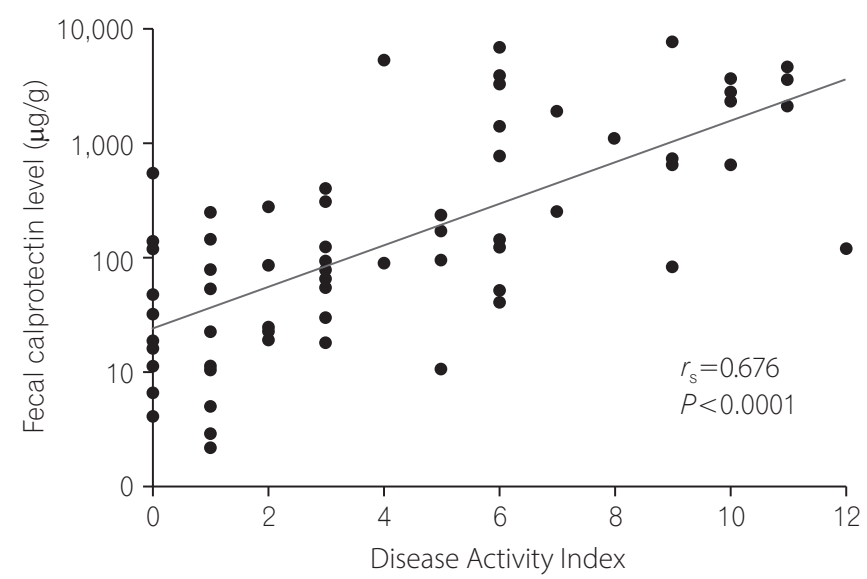

involvement, 22 had L3 (ileocolon) involvement, and 2 had some other involvement.

\section{FCP Levels in UC Patients}

FCP levels in UC patients strongly correlated with the DAI score $\left(r_{s}=0.676, P<0.0001\right)$ and MES $\left(r_{s}=0.677, P<0.0001\right)$ (Fig. 2). FCP levels in the inactive UC group $(\mathrm{n}=27)$ ranged from 6.7 to $1,735.4 \mu \mathrm{g} / \mathrm{g}$ (median, $72.7 \mu \mathrm{g} / \mathrm{g}$ ), while FCP levels in the active UC group ( $\mathrm{n}=37)$ ranged from 34.2 to $24,422.1 \mu \mathrm{g} /$ $\mathrm{g}$ (median, $798.1 \mu \mathrm{g} / \mathrm{g})(P<0.0001)$ (Fig. 3). Even in the inactive UC group, FCP levels were significantly higher than in HCs (n=64), ranging from 6.7 to $1,735.4 \mu \mathrm{g} / \mathrm{g}$ (median, 72.7 $\mu \mathrm{g} / \mathrm{g} ; P=0.0068)$.

FCP levels were significantly higher in active E2 or E3 pa-

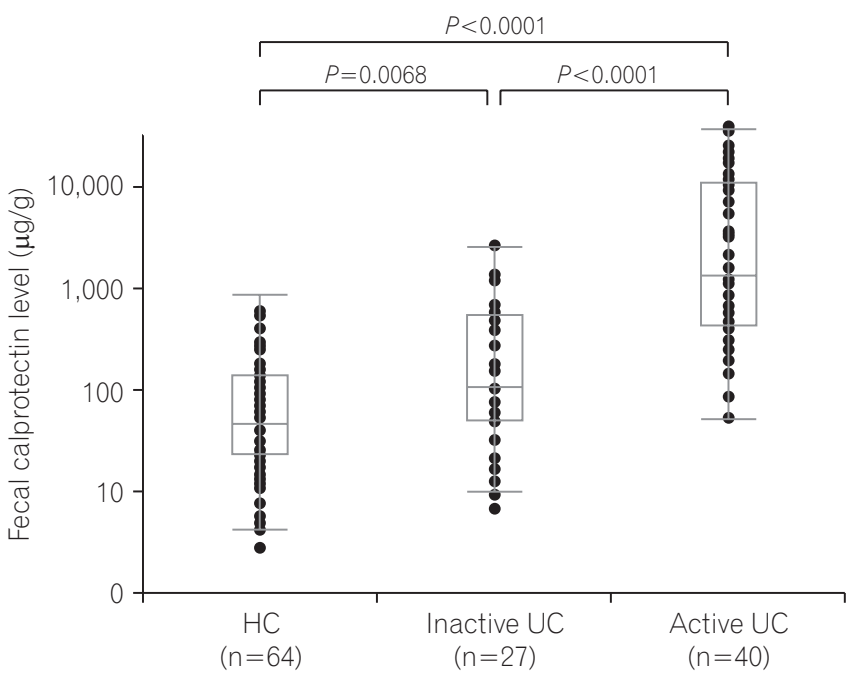

Fig. 3. Fecal calprotectin levels in healthy control $(\mathrm{HC})$ subjects, patients with inactive UC, and patients with active UC.

B

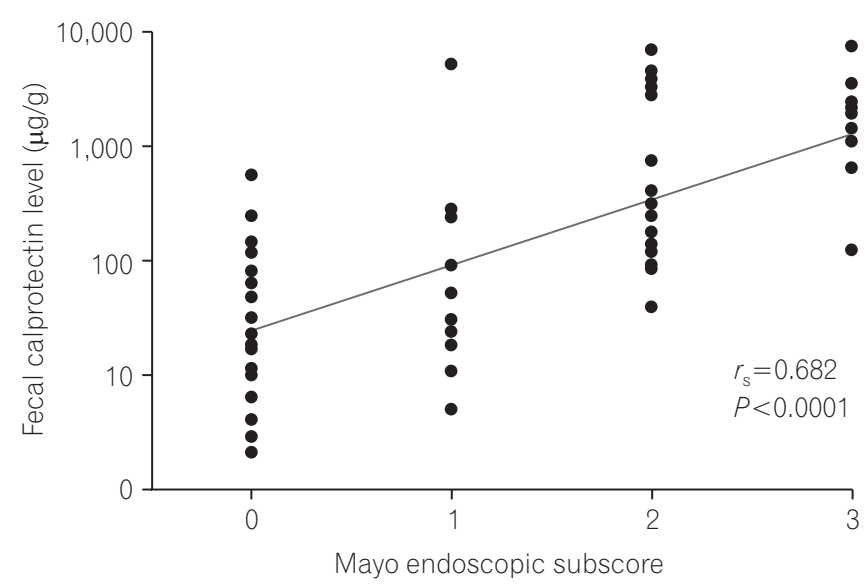

Fig. 2. Relationship between fecal calprotectin levels and Disease Activity Index (A) and Mayo endoscopic subscore (B) for patients with UC. 
tients than in inactive UC patients $(P=0.0004, P<0.0001)$, but they were not significantly higher in active $\mathrm{E} 1$ patients than in inactive UC patients $(P=0.557)$.

\section{1) Discriminative Ability between Inactive UC and Active UC}

In terms of discriminative ability between inactive UC and active UC, the AUC was 0.845 (cutoff value $=161.3 \mu \mathrm{g} / \mathrm{g}$, sensitivity $=89.2 \%$, specificity $=63.0 \%)$.

\section{2) Discriminative Ability between Each Adjacent Stage as Assessed by MES}

Results for discriminative ability between each adjacent stage as assessed by MES are presented in Table 2. In particular, the AUC between MES 1 and 2 was 0.842 (optimal cutoff value $=261.4 \mu \mathrm{g} / \mathrm{g}$, sensitivity $=94.1 \%$, specificity $=68.8 \%$ ), suggesting the usefulness of FCP for discrimination between these 2 stages. While the AUC between MES 0 and 1 was 0.578 (optimal cutoff value $=34.2 \mu \mathrm{g} / \mathrm{g}$, sensitivity $=93.8 \%$, specificity $=30.0 \%)$.

\section{FCP Levels in CD Patients}

There were 28 inactive CD patients and 18 active CD patients (mild activity $[150<\mathrm{CDAI}<220]$ in 13 patients, moderate $[220<\mathrm{CDAI}<450]$ in 4 , and severe $[\mathrm{CDAI}>450]$ in 1$)$. FCP levels ranged from 2.0 to $1,280.8 \mu \mathrm{g} / \mathrm{g}$ (median, $143.6 \mu \mathrm{g} / \mathrm{g}$ ) in inactive CD patients and from 15.3 to $6,911.8 \mu \mathrm{g} / \mathrm{g}$ (median, $482.3 \mu \mathrm{g} / \mathrm{g})$ in active CD patients $(P=0.0579)$. Even in the inactive CD group, FCP levels were significantly higher than in HCs $(P<0.0001)$ (Fig. 4A). For all CD patients, CDAI tended to correlate with the FCP level $\left(r_{s}=0.283, P=0.0565\right)$ (Fig. 4B). FCP levels ranged from 15.3 to $6,911.8 \mu \mathrm{g} / \mathrm{g}$ (median, $292.1 \mu \mathrm{g} / \mathrm{g}$ ) in CD patients with mild activity and from 36.9 to $5,102.2 \mu \mathrm{g} / \mathrm{g}$ (median, $1,585.7 \mu \mathrm{g} / \mathrm{g}$ ) in CD patients with moderate to severe activity $(P=0.4002)$.

FCP levels did not differ significantly between patients with inactive $\mathrm{CD}$ and patients with active $\mathrm{L} 1$ involvement ( $P=0.2587)$, active $\mathrm{L} 2$ involvement $(P=0.1575)$, or active L3 involvement $(P=0.3200)$, probably because of the small number of participants.

In terms of discriminative ability between inactive $\mathrm{CD}$ and

Table 2. Discriminative Ability between Each Adjacent Stage as Assessed by MES for UC Subjects

\begin{tabular}{lcccc}
\hline & HC vs. MESO & MES0 vs. MES1 & MES1 vs. MES2 & MES2 vs. MES3 \\
\hline AUC & 0.655 & 0.578 & 0.842 & 0.695 \\
Optimal cutoff value $(\mu \mathrm{g} / \mathrm{g})$ & 31.3 & 34.2 & 261.4 & $2,064.9$ \\
Sensitivity $(\%)$ & 51.6 & 93.8 & 94.1 & 81.8 \\
Specificity $(\%)$ & 80.0 & 30.0 & 68.8 & 64.7 \\
\hline
\end{tabular}

MES, Mayo endoscopic subscore; $\mathrm{HC}$, healthy control; $\mathrm{AUC}$, area under the receiver operating characteristics.

A

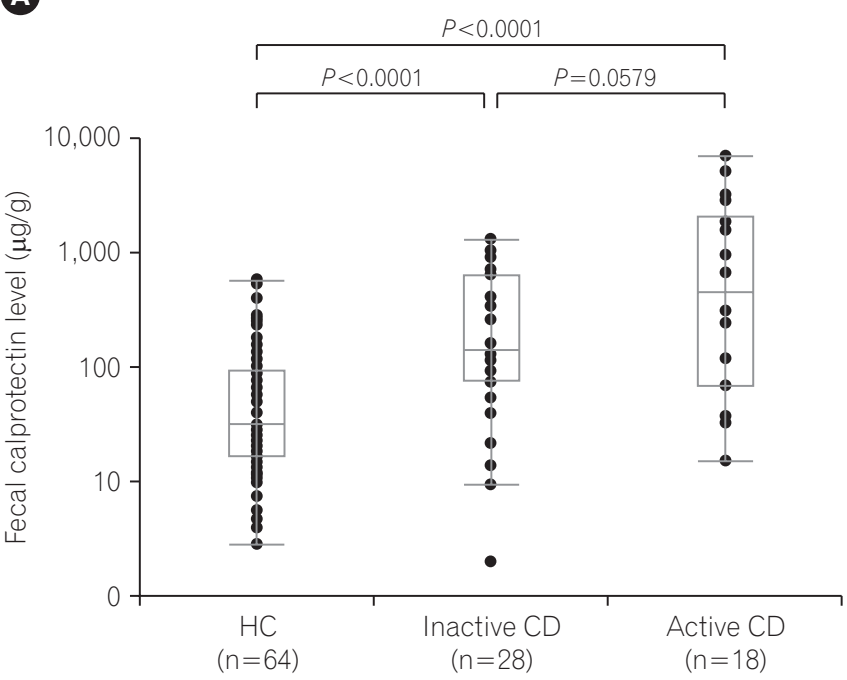

B

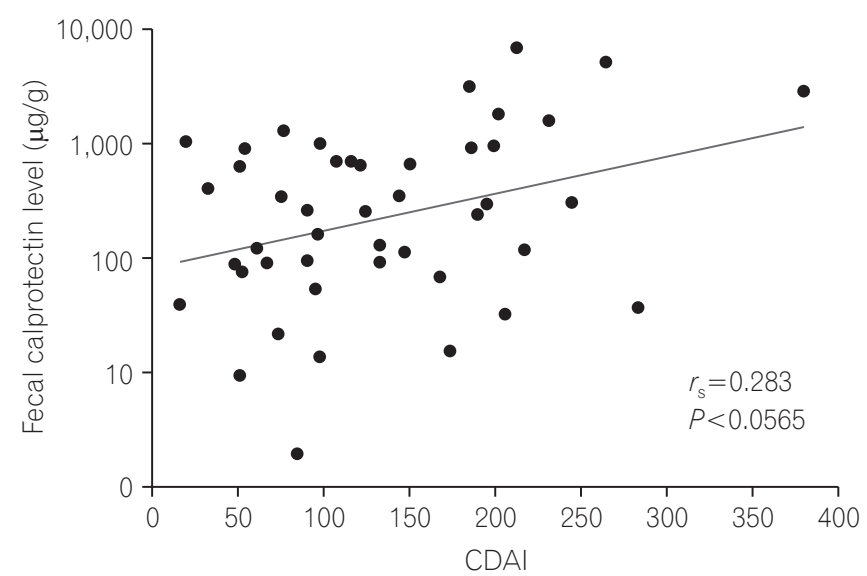

Fig. 4. (A) Fecal calprotectin (FCP) levels in healthy control $(H C)$ subjects, patients with inactive $C D$, and patients with active $C D$. (B) Relationship between FCP levels and CDAI in CD patients. 
Table 3. Relationship between Fecal Calprotectin Level and Laboratory Parameters in UC and CD Patients

\begin{tabular}{lrrrrr}
\hline & \multicolumn{3}{c}{ UC } & & \multicolumn{2}{c}{ CD } \\
\cline { 2 - 3 } \cline { 5 - 6 } & \multicolumn{1}{c}{$r_{s}$} & $P$-value & & $r_{s}$ & $P$-value \\
\hline WBC & 0.347 & 0.0227 & & 0.280 & 0.0936 \\
Hemoglobin & -0.326 & 0.0329 & -0.234 & 0.1635 \\
Platelet count & 0.492 & 0.0008 & 0.522 & 0.0009 \\
Total protein & -0.367 & 0.0182 & -0.094 & 0.5930 \\
Serum albumin & -0.698 & $<0.0001$ & -0.345 & 0.0426 \\
CRP & 0.753 & $<0.0001$ & 0.416 & 0.0116 \\
ESR & 0.592 & 0.0007 & 0.487 & 0.0343 \\
\hline
\end{tabular}

WBC, white blood cells.

active CD, the AUC was 0.643 (cutoff value $=934.3 \mu \mathrm{g} / \mathrm{g}$, sensitivity $=44.4 \%$, specificity=89.3\%).

\section{Relationship between Laboratory Parameters and FCP Levels in UC and CD Patients}

In UC patients, white blood cell count, hemoglobin, platelet count, total protein, serum albumin, CRP and ESR significantly correlated with the FCP level. In CD patients, platelet count, albumin, CRP, and ESR significantly correlated with the FCP level. The corresponding $r_{s}$ values and $P$-values are shown in Table 3.

\section{FCP Levels in HCs According to the Presence or Absence of Abdominal Symptoms}

The median FCP level in the PASG (29.6 $\mu \mathrm{g} / \mathrm{g}$; range, $4.7-$ $280.5 \mu \mathrm{g} / \mathrm{g} ; \mathrm{n}=21$ ) was not significantly higher than that in the NASG $(55.0 \mu \mathrm{g} / \mathrm{g}$; range, $2.8-568.3 \mu \mathrm{g} / \mathrm{g} ; \mathrm{n}=43)(P=0.208)$.

\section{DISCUSSION}

The findings of the present study confirmed the usefulness of FCP measurements for adult Japanese patients with IBD. We revealed that FCP levels correlated well with the DAI score in UC subjects $\left(r_{s}=0.676, P<0.0001\right)$ and tended to correlate with the CDAI in CD subjects $\left(r_{s}=0.283, P=0.0565\right)$. Consistent with previous reports, FCP levels well discriminated between active and inactive UC (optimal cutoff point $=161.3$ $\mu \mathrm{g} / \mathrm{g}, \mathrm{AUC}=0.845$ ) and active and inactive CD (optimal cutoff point=934.3 $\mu \mathrm{g} / \mathrm{g}, \mathrm{AUC}=0.643$ ), and numerous laboratory parameters (platelet count, albumin, CRP and ESR). ${ }^{14-26}$ The lack of a significant difference in FCP levels $(P=0.1393)$ between patients with UC and CD may be due to differences in their disease severities $(57.8 \%$ [37/64] for active UC patients vs. 39.1\% [18/46] for active CD patients).

A recent clinicians' guide to the use of FCP for identifying and monitoring disease activity in IBD patients recommends the following: (1) In patients with FCP levels $<50-100$ $\mu \mathrm{g} / \mathrm{g}$, quiescent disease is likely and continuation of current therapy is recommended; (2) In patients with FCP levels $>100-250 \mu \mathrm{g} / \mathrm{g}$, inflammation is possible and further examination such as colonoscopy is needed to identify inflammatory lesions; (3) In patients with FCP levels > $250 \mu \mathrm{g} / \mathrm{g}$, active inflammatory lesions are likely and therapies to regulate inflammation should be considered. ${ }^{24}$ These recommendations are based on data of Western IBD patients. ${ }^{24}$ Based on the optimal cutoff points determined in the present study, these recommendations are well validated in adult Japanese IBD patients. Discrimination between inactive and active IBD is highly important clinically. Noteworthy is that in UC patients with FCP levels $<50 \mu \mathrm{g} / \mathrm{g}$ (judged as quiescent disease), one patient had an elevated DAI score ( 5 points) and the Montreal classification in this case was E1 (ulcerative proctitis). In UC patients with a limited disease extent, however, lower FCP levels do not always suggest quiescent disease. In fact, FCP levels were significantly higher in patients with active E2 or E3 involvement than in those with inactive UC, but not higher in patients with active E1 involvement compared with those with inactive UC in the present study.

In our study, the FCP levels widely ranged from 6.7 to $24,422.1 \mu \mathrm{g} / \mathrm{g}$ in UC patients and from 2.0 to $6,911.8 \mu \mathrm{g} / \mathrm{g}$ in CD patients. Several factors, including disease activity, disease extent, complications, comorbidity, age, and error of measurement by ELISA, affect FCP levels. One possible reason for these results is that the FCP level may be partly associated with the IBD disease extent, regardless of the disease severity. ${ }^{27}$ Our findings that the median FCP level was $134.6 \mu \mathrm{g} / \mathrm{g}$ in inactive UC patients with E3 (extensive UC), and $50.5 \mu \mathrm{g} / \mathrm{g}$ in patients with other IBD types may support this hypothesis.

Objective monitoring, including endoscopic assessment, is essential to optimize treatment. In UC patients, most clinical trials have used the threshold between mucosal healing (MES 0 or 1 ) and non-mucosal healing (MES 2 or 3 ) as a guide for treatment. Our FCP measurement was useful for differentiating between mucosal healing and non-mucosal healing (MES 1 vs. MES 2: AUC=0.842). On the other hand, the AUC between MES 0 and 1 was 0.578 (sensitivity=93.8\%, specificity $=30.0 \%$ ) in our UC patients. The median FCP values in patients with MES 0, 1, 2, and 3 were 85.1, 127.7, 798.1 , and $4,534.8 \mu \mathrm{g} / \mathrm{g}$, respectively. The slight difference in median FCP levels in MES 0 and 1, and the remarkable dif- 
ference in median FCP levels in MED 1 and 2 may account for the present results.

A major finding in our study is that the usefulness of the Calprotectin ELISA Kit (Calprotectin Mochida ${ }^{\circledR}$, tested by sandwich enzyme immunoassay using a monoclonal antibody) for measuring FCP levels was validated in Japanese IBD patients. Kawashima et al. ${ }^{27}$ (Japanese investigators) reported that FCP levels determined using quantitative ELISA (PhiCal ${ }^{\circledR}$, Immundiagnostik, Bensheim, Germany) correlated with both the extent of the affected mucosa and disease severity in UC patients. In the study by Yamaguchi et al. ${ }^{29}$ (Japanese investigators), FCP was tested by a fluorescence enzyme immunoassay that differs from our current testing method. A previous study group reported the predictive superiority of monoclonal testing of calprotectin, but their study subjects were Western patients. ${ }^{32}$ Aomatsu et al. ${ }^{33,34}$ previously reported FCP levels determined using a monoclonal antibody in pediatric IBD patients. There has been no previous investigation of FCP levels using a monoclonal antibody in adult IBD patients. Li et al.$^{35}$ reported that FCP levels were higher in healthy children than in healthy adults. Importantly, the present findings confirm the usefulness of our testing method for FCP by sandwich enzyme immunoassay using a monoclonal antibody in adult Japanese IBD patients.

Amcoff et al. ${ }^{38}$ recently reported the results of comparison with different FCP assays, 2 ELISA (BÜHLMANN and Immunodiagnostik) and 1 automated fluoroimmunoassay (Phadia). They clearly showed the optimal threshold for discriminating between clinical remission and active disease in IBD is assay specific and especially the BÜHLMANN assay improved compared to the other 2 assays when the cutoff for positive test was increased. Therefore, we thought the FCP assays of BÜHLMANN that we used in the present study has the superiority. And there were few previous reports with BÜHLMANN assays in Asian patients with IBD. Lin et al. ${ }^{39}$ investigated the correlation between FCP levels and CRP titers or the Ulcerative Colitis Endoscopic Index of Severity in 52 Taiwanese UC patients, 36 Taiwanese CD patients and 11 controls. But they used only the rapid testing (Quantum Blue Calprotectin High Range Rapid Test; BÜHLMANN laboratories AG, Schonenbuch, Switzerland). Fukunaga et al. ${ }^{40}$ reported the results of FCP levels in 113 Japanese UC patients, 42 Japanese CD patients and 96 controls by using both of conventional ELISA (BÜHLMANN) and rapid testing (Quantum Blue Calprotectin High Range Rapid Test; BÜHLMANN). However, they did not evaluate the correlation between FCP levels and endoscopic scores of UC.
IBS is a functional gastrointestinal disorder with a high worldwide population prevalence. Clinical symptoms for IBS patients are abdominal pain or abdominal discomfort, bowel movement irregularities, and abdominal bloating, as well as other somatic, visceral, and psychiatric disorders without organic abnormality. ${ }^{41}$ In the current analysis, the difference between the PASG and NASG was not significant with regard to the FCP levels. FCP level may not be useful for distinguishing between IBS and healthy persons without abdominal symptoms, although this is beyond the scope of the current analysis. The median FCP level in the PASG (29.6 $\mu \mathrm{g} / \mathrm{g}$ ) was low. Thus, assessment of FCP levels may be useful for differential diagnosis between active IBD and IBS among suspected IBD patients. In the present study, the FCP levels in both inactive UC and inactive CD patients were significantly higher than those in HCs.

A limitation of the present study is that we did not evaluate the relationship between FCP levels and clinical outcomes, such as disease progression. And the disease activity of IBD patients recruited in this study was low mainly. However, we think the major part of utilities for FCP measurement in IBD patients are in lower activity phase because we can assess the activity in higher activity phase by the patients' symptoms without FCP measurement. Another limitation is that the number of subjects in our study cohort, especially CD patients, was relatively small for analysis. We did not evaluate the endoscopic score of CD because small number of recruited CD patients could be assessed for the endoscopic score of CD. Larger prospective studies are necessary. In this study, however, the usefulness of measuring FCP levels by our testing method using a monoclonal antibody in adult Japanese IBD patients was well confirmed in a prospective multicenter trial.

In conclusion, our testing method using a monoclonal antibody to measure FCP levels was well validated and could differentiate between patients with IBD and HCs. FCP may be a useful biomarker for objective assessment of disease activity in adult Japanese IBD patients, especially those with UC.

\section{FINANCIAL SUPPORT}

This work was supported in part by Health and Labor Sciences Research Grants for research on intractable diseases from the Ministry of Health, Labor, and Welfare of Japan. 


\section{CONFLICT OF INTEREST}

The authors have the following financial conflicts of interest regarding this manuscript. Shiro Nakamura, KW, HN, and AA (Sanyo Chemical Industries, Ltd. and Mochida Pharmaceutical Co., Ltd.).

\section{AUTHOR CONTRIBUTION}

Study conception and design: Shiro Nakamura, HH, KW, HN, and AA; data acquisition: Shiro Nakamura, HI, MI, TY, MM, HO, JO, YM, KW, HN, and AA; data analysis and interpretation: Shiro Nakamura, HN, RO, SNi, MI, KW, and HH; drafting the article: Shiro Nakamura, HN, RO, SNi, MI, and KW.

\section{REFERENCES}

1. Torres J, Mehandru S, Colombel JF, Peyrin-Biroulet L. Crohn's disease. Lancet 2017;389:1741-1755.

2. Loftus EV Jr. Crohn's disease: why the disparity in mortality? Gut 2006;55:447-449.

3. Selinger CP, Leong RW. Mortality from inflammatory bowel diseases. Inflamm Bowel Dis 2012;18:1566-1572.

4. Cosnes J, Gower-Rousseau C, Seksik P, Cortot A. Epidemiology and natural history of inflammatory bowel diseases. Gastroenterology 2011;140:1785-1794.

5. Ueno F, Matsui T, Matsumoto T, et al. Evidence-based clinical practice guidelines for Crohn's disease, integrated with formal consensus of experts in Japan. J Gastroenterol 2013;48:31-72.

6. Feuerstein JD, Cheifetz AS. Ulcerative colitis: epidemiology, diagnosis, and management. Mayo Clin Proc 2014;89:1553-1563.

7. Walsh AJ, Bryant RV, Travis SP. Current best practice for disease activity assessment in IBD. Nat Rev Gastroenterol Hepatol 2016; 13:567-579.

8. Ket SN, Palmer R, Travis S. Endoscopic disease activity in inflammatory bowel disease. Curr Gastroenterol Rep 2015;17:50. doi: 10.1007/s11894-015-0470-0.

9. Menees S, Higgins P, Korsnes S, Elta G. Does colonoscopy cause increased ulcerative colitis symptoms? Inflamm Bowel Dis 2007; 13:12-18.

10. Däbritz J, Musci J, Foell D. Diagnostic utility of faecal biomarkers in patients with irritable bowel syndrome. World J Gastroenterol 2014;20:363-375.

11. Panes J, Jairath V, Levesque BG. Advances in use of endoscopy, radiology, and biomarkers to monitor inflammatory bowel diseases. Gastroenterology 2017;152:362-373.e3.
12. Lopez RN, Leach ST, Lemberg DA, Duvoisin G, Gearry RB, Day AS. Fecal biomarkers in inflammatory bowel disease. J Gastroenterol Hepatol 2017;32:577-582.

13. Manceau H, Chicha-Cattoir V, Puy H, Peoc'h K. Fecal calprotectin in inflammatory bowel diseases: update and perspectives. Clin Chem Lab Med 2017;55:474-483.

14. Kopylov U, Yung DE, Engel T, et al. Fecal calprotectin for the prediction of small-bowel Crohn's disease by capsule endoscopy: a systematic review and meta-analysis. Eur J Gastroenterol Hepatol 2016;28:1137-1144.

15. Mosli MH, Zou G, Garg SK, et al. C-reactive protein, fecal calprotectin, and stool lactoferrin for detection of endoscopic activity in symptomatic inflammatory bowel disease patients: a systematic review and meta-analysis. Am J Gastroenterol 2015;110:802-819.

16. Henderson P, Anderson NH, Wilson DC. The diagnostic accuracy of fecal calprotectin during the investigation of suspected pediatric inflammatory bowel disease: a systematic review and meta-analysis. Am J Gastroenterol 2014;109:637-645.

17. Ma C, Lumb R, Walker EV, et al. Noninvasive fecal immunochemical testing and fecal calprotectin predict mucosal healing in inflammatory bowel disease: a prospective cohort study. Inflamm Bowel Dis 2017;23:1643-1649.

18. van Rheenen PF, Van de Vijver E, Fidler V. Faecal calprotectin for screening of patients with suspected inflammatory bowel disease: diagnostic meta-analysis. BMJ 2010;341:c3369. doi: 10.1136/bmj.c3369.

19. Scaioli E, Scagliarini M, Cardamone C, et al. Clinical application of faecal calprotectin in ulcerative colitis patients. Eur J Gastroenterol Hepatol 2015;27:1418-1424.

20. Taghvaei T, Maleki I, Nagshvar F, et al. Fecal calprotectin and ulcerative colitis endoscopic activity index as indicators of mucosal healing in ulcerative colitis. Intern Emerg Med 2015;10:321328.

21. Lobatón T, López-García A, Rodríguez-Moranta F, Ruiz A, Rodríguez L, Guardiola J. A new rapid test for fecal calprotectin predicts endoscopic remission and postoperative recurrence in Crohn's disease. J Crohns Colitis 2013;7:e641-e651. doi: 10.1016/j.crohns. 2013.05.005.

22. Lobatón T, Rodríguez-Moranta F, Lopez A, Sánchez E, Rodríguez-Alonso L, Guardiola J. A new rapid quantitative test for fecal calprotectin predicts endoscopic activity in ulcerative colitis. Inflamm Bowel Dis 2013;19:1034-1042.

23. Schoepfer AM, Beglinger C, Straumann A, et al. Fecal calprotectin more accurately reflects endoscopic activity of ulcerative colitis than the Lichtiger Index, C-reactive protein, platelets, hemoglobin, and blood leukocytes. Inflamm Bowel Dis 2013;19: 332-341. 
24. Bressler B, Panaccione R, Fedorak RN, Seidman EG. Clinicians' guide to the use of fecal calprotectin to identify and monitor disease activity in inflammatory bowel disease. Can J Gastroenterol Hepatol 2015;29:369-372.

25. Tibble JA, Sigthorsson G, Foster R, Forgacs I, Bjarnason I. Use of surrogate markers of inflammation and Rome criteria to distinguish organic from nonorganic intestinal disease. Gastroenterology 2002;123:450-460.

26. Ikhtaire S, Shajib MS, Reinisch W, Khan WI. Fecal calprotectin: its scope and utility in the management of inflammatory bowel disease. J Gastroenterol 2016;51:434-446.

27. Kawashima K, Ishihara S, Yuki T, et al. Fecal calprotectin level correlated with both endoscopic severity and disease extent in ulcerative colitis. BMC Gastroenterol 2016;16:47. doi: 10.1186/ s12876-016-0462-Z.

28. Inokuchi T, Kato J, Hiraoka S, et al. Fecal immunochemical test versus fecal calprotectin for prediction of mucosal healing in Crohn's disease. Inflamm Bowel Dis 2016;22:1078-1085.

29. Yamaguchi S, Takeuchi Y, Arai K, et al. Fecal calprotectin is a clinically relevant biomarker of mucosal healing in patients with quiescent ulcerative colitis. J Gastroenterol Hepatol 2016;31:9398.

30. Hanai H, Takeuchi K, Iida T, et al. Relationship between fecal calprotectin, intestinal inflammation, and peripheral blood neutrophils in patients with active ulcerative colitis. Dig Dis Sci 2004;49:1438-1443.

31. Yang SK, Hong M, Oh H, et al. Identification of loci at 1q21 and $16 q 23$ that affect susceptibility to inflammatory bowel disease in Koreans. Gastroenterology 2016;151:1096-1099.e4.

32. Burri E, Manz M, Rothen C, Rossi L, Beglinger C, Lehmann FS. Monoclonal antibody testing for fecal calprotectin is superior to polyclonal testing of fecal calprotectin and lactoferrin to identify organic intestinal disease in patients with abdominal discomfort. Clin Chim Acta 2013;416:41-47.
33. Aomatsu T, Yoden A, Matsumoto K, et al. Fecal calprotectin is a useful marker for disease activity in pediatric patients with inflammatory bowel disease. Dig Dis Sci 2011;56:2372-2377.

34. Aomatsu T, Imaeda H, Matsumoto K, et al. Faecal chitinase 3-like-1: a novel biomarker of disease activity in paediatric inflammatory bowel disease. Aliment Pharmacol Ther 2011;34: 941-948.

35. Li F, Ma J, Geng S, et al. Fecal calprotectin concentrations in healthy children aged 1-18 months. PLoS One 2015;10:e0119574. doi: 10.1371/journal.pone.0119574.

36. Sutherland LR, Martin F, Greer S, et al. 5-Aminosalicylic acid enema in the treatment of distal ulcerative colitis, proctosigmoiditis, and proctitis. Gastroenterology 1987;92:1894-1898.

37. Silverberg MS, Satsangi J, Ahmad T, et al. Toward an integrated clinical, molecular and serological classification of inflammatory bowel disease: report of a Working Party of the 2005 Montreal World Congress of Gastroenterology. Can J Gastroenterol 2005;19 Suppl A:5A-36A.

38. Amcoff K, Stridsberg M, Lampinen M, Magnuson A, Carlson M, Halfvarson J. Clinical implications of assay specific differences in $\mathrm{f}$-calprotectin when monitoring inflammatory bowel disease activity over time. Scand J Gastroenterol 2017;52:344-350.

39. Lin WC, Wong JM, Tung CC, et al. Fecal calprotectin correlated with endoscopic remission for Asian inflammatory bowel disease patients. World J Gastroenterol 2015;21:13566-13573.

40. Fukunaga S, Kuwaki K, Mitsuyama K, et al. Detection of calprotectin in inflammatory bowel disease: fecal and serum levels and immunohistochemical localization. Int J Mol Med 2018; 41:107-118.

41. Enck P, Aziz Q, Barbara G, et al. Irritable bowel syndrome. Nat Rev Dis Primers 2016;2:16014. doi: 10.1038/nrdp.2016.14. 